\title{
How Sensor, Signal, and Imaging Informatics May Impact Patient Centered Care and Care Coordination
}

\author{
S. Voros', A. Moreau-Gaudry ${ }^{1,2}$, Section Editors for the IMIA Yearbook Section on Sensor, \\ Signal and Imaging Informatics \\ 1 Univ. Grenoble Alpes, TIMC-IMAG; CNRS, INSERM, TIMC-IMAG, F-38000, Grenoble, France \\ 2 Inserm CIC 1406; CHU Grenoble, Pôle Santé Publique, CIC-IT, F-38000, Grenoble, France
}

\section{Summary}

Objective: This synopsis presents a selection for the IMIA (International Medical Informatics Association) Yearbook 2015 of excellent research in the brood field of Sensor, Signal, and Imaging Informatics published in the year 2014, with a focus on patient centered care coordination.

Methods: The two section editors performed a systematic initial selection and a double blind peer review process to select a list of candidate best papers in the domain published in 2014 , from the PubMed and Web of Science databases. A set of MeSH keywords provided by experts was used. This selection was peer-reviewed by external reviewers.

Results: The review process highlighted articles illustrating two current trends related to care coordination and patient centered care: the enhanced capacity to predict the evolution of a disease based on patient-specific information can impact care coordination; similarly, better perception of the patient and his treatment could lead to enhanced personalized care with a potential impact on care coordination.

Conclusions: This review shows the multiplicity of angles from which the question of patient-centered care can be addressed, with consequences on care coordination that will need to be confirmed and demonstrated in the future.

\section{Keywords}

Medicine personalization, care coordination, innovative technologies, biomedical imaging, patient centered modelling

Yearb Med Inform 2015, 10:102-5

http://dx.doi.org/10.15265//Y-2015-025

Published online August 13, 2015

\section{Introduction}

The selection is composed of papers addressing innovative approaches in the field of sensor, signal, and imaging informatics with a special focus on patient centered care and care coordination. The selection was performed within PubMed and Web of Sciences databases, according to a generic MesH keywords query provided by experts.

\section{Results}

We reproduced the automatic selection process presented in the 2014 Yearbook, which resulted in a large amount of publications: 1233 articles (vs. 1361 in 2013), illustrating again the intense activity in the field. We pre-selected 22 articles as candidate best paper in the double blind review process. Among these, 13 articles were selected. The five best papers were selected thanks to an external review process.

The selection shows a general tendency centered on better understanding of the patient and his treatment, which, in term could impact care coordination. This better understanding can be achieved by a) the capacity to predict the patient-specific evolution of a disease and b) the capacity to better perceive information on the patient and the care delivered.

\section{Discussion}

\section{Prediction of Patient Specific Evolution of a Disease}

Prediction models to characterize the evolution of a disease have sparked interest for the past few years, aiming at better patient care. In the more recent works, those approaches are personalized by taking into account the patient's clinical measurements. For instance, in the oncology field, [1] reports a method for the prediction of lung tumor evolution that uses functional imaging to personalize a mathematical model (partial differential equations) describing the evolution of tumor cell density. The method was evaluated on a synthetic dataset and on PET data from 7 non-small cell lung carcinoma patients. Evaluation on patient data shows a good correlation between estimated and real evolution of the disease with four indices (e.g. $76.5+/-16.74 \%$ of volume concordance). Similar work is presented in [2] involving temporal sequences of CT scans, with smaller scale evaluation (1 real clinical case). In addition, [3] links the mathematical model for tumor growth, calibrated from CT and PET images, with an hyperelastic biomechanical model. This model constrains the way the tumor is going to develop. Their approach is validated on 8 patients images with pancreatic neuroendocrine tumors (relative volume difference of $13.8+/-6.6 \%$ ). Prediction 
approaches can also be used in other medical fields, for instance, for estimating the survival rate of ICU patients [4].

The group of articles presented above may directly impact patients care coordination. Conversely the next four paragraphs will focus on a better understanding of the patient and his environment that may also indirectly impact patient care coordination in the future. Such understanding is tackled through novel imaging devices and image-analysis approaches, innovative ancillary surgical material, aand the modelling of healt-actors and surgical environment interactions.

\section{Novel Imaging Devices and Image- analysis Approaches}

Two reviews have been identified this year on disruptive imaging technologies that could provide tremendous intra-operative information at different temporal and morphologic scales than available today. [5] presents the concept and current and future clinical applications of ultrafast ultrasound imaging, that offer new perspectives: shearwave elastography with the possibility to quantify tissue biomechanical properties; ultrafast Doppler imaging allowing to both image and quantify blood flow; ultrafast imaging of contrast agents with applications for non-destructive real-time micro-bubbles imaging; and most fascinatingly, the possibility to perform functional ultrasound imaging. The review [6] focuses on intra-operative optical imaging that could offer the surgeon the real-time capacity to visualize diseased and healthy tissue at micrometer scale (cellular) or below (molecular).

In addition to these new sensors, new signal-analysis techniques are currently developed to highlight information contained in current clinically available sensors, but inaccessible until now. For instance, in the field of gastrointestinal tract lesions identifications, a challenge consists in manually labelling large quantity of images acquired by wireless capsule endoscopy. [7] proposes a new method to automatically and rapidly detect bleeding in such images. In [8], the authors answer the challenge of detecting vascular structures during laparoscopic

Table 1 Best paper selection of articles for the IMIA Yearbook of Medical Informatics 2015 in the section 'Sensors, Signal and Imaging Informatics'. The articles are listed in alphabetical order of the first author's surname.

\section{Section}

Sensors, Signal and Imaging Informatics

- Amir-Khalili A, Peyrat J-M, Abinahed J, Al-Alao 0, Al-Ansari A, Hamarneh G, Abugharbieh R. Auto localization and segmentation of occluded vessels in robot-assisted partial nephrectomy. Med Image Comput Comput Assist Interv 2014;17(1):407-14.

- Mi H, Petitiean C, Dubray B, Vera P, Ruan S. Prediction of lung tumor evolution during radiotherapy in individual patients with PET. IEEE Trans Med Imaging 2014 Apr;33(4):995-1003.

- Pheiffer TS, Thompson RC, Rucker DC, Simpson AL, Miga MI. Model-based correction of tissue compression for tracked ultrasound in soft tissue image-guided surgery. Ultrasound Med Biol 2014 Apr;40(4):788-803.

- Quellec G, Charrière K, Lamard M, Droueche Z, Roux C, Cochener B, Cazuguel G. Real-time recognition of surgical tasks in eye surgery videos. Med Image Anal 2014 Apr;18(3):579-90.

- Tanter M, Fink M. Ultrafast imaging in biomedical ultrasound. IEEE Trans Ultrason Ferroelectr Freq Control 2014 Jan;61(1):102-19.

surgery by an image-based analysis solution. Indeed such structures have to be preserved to avoid surgical complications. The approach is adapted from phase-based video magnification and allows to measure subtle periodic motions that are not accessible by the human eye. Finally, from a more prospective point of view, and on the contrary to conventional approaches that try to eliminate the noise contained in the acquired data, [9] incorporates it in a new information processing framework, allowing better extraction of relevant information contained in images. This framework has been in particular evaluated for the detection of lesions in mammogram images.

\section{Innovative Ancillary Surgical Material}

In the general context of better understanding and treating the patient, innovative ancillary surgical materials now give the possibility to access patient specific data, from which patients care can be personalized. This year's review process highlighted two different approaches focused on bone surgery: [10] uses rapid prototyping to construct bone templates for mandibular reconstructive surgery. These templates are designed from patient-specific pre-operative models build with well-known medical image processing tools. The approach was validated successfully on 15 clinical cases. Similarly, in response to the occlusion problem inherent to optical navigation systems, [11] proposes a new localizing system based on accelerometers, in order to quantitatively access the static and dynamic function of the knee joint. Such personalized information is essential to adapt intra-operatively the surgical gesture according to the patient's anatomy.

\section{Health-actors and Surgical Environment Interactions Modelling}

One of the keys for better treating a patient is also to understand the interactions between the patient and his environment during a surgical procedure, from which personalized interaction models can be developed. This can involve the ability to automatically identify in real-time surgical tasks, as illustrated by [12] in the context of retinal surgery. This work suggests perspectives in intra-operative mentoring or surgical devices supervision according to the current surgery process. [13] reports a new approach allowing the intra-operative correction of Ultrasound images, to take into account the effect of the ultrasound probe's compression on the patient's tissue. This work offers perspectives in image-guided surgeries, by reducing the registration errors with CT or MRI pre-operative models. Combining such approaches would probably allow to apprehend even better the interactions between the clinician's instruments and his patient, for task specific and patient-specific personalized care. 


\section{Conclusion \& Outlook}

This review illustrates the multiplicity of angles from which the question of patient-centered care can be addressed, with a potential impact on care coordination, which still needs to be demonstrated from a public health point of view. Along with the big data field that is also coming to maturity, as reviewed last year, we can suspect that new modalities of patient care will emerge, in the respect of health-policies constraints.

\section{References}

1. Mi H, Petitjean C, Dubray B, Vera P, Ruan S. Prediction of lung tumor evolution during radiotherapy in individual patients with PET. IEEE Trans Med Imaging 2014 Apr;33(4):995-1003.

2. Colin T, Cornelis F, Jouganous J, Martin M, Saut O. Patient specific image driven evaluation of the aggressiveness of metastases to the lung. Med Image Comput Comput Assist Interv 2014;17(1):553-60.

3. Wong KCL, Summers RM, Kebebew E, Yao J. Tumor growth prediction with hyperelastic biomechanical model, physiological data fusion, and nonlinear optimization. Med Image Comput Comput Assist Interv 2014;17(2):25-32.

4. Hsieh Y-Z, Su M-C, Wang C-H, Wang P-C. Prediction of survival of ICU patients using computational intelligence. Comput Biol Med 2014;47:13-9.

5. Tanter M, Fink M. Ultrafast imaging in biomedical ultrasound. IEEE Trans Ultrason Ferroelectr Freq Control 2014 Jan;61(1):102-19.

6. Hsu M, Gupta M, Su L-M, Liao JC. Intraoperative optical imaging and tissue interrogation during urologic surgery. Curr Opin Urol 2014;24(1):66-74.

7. Fu Y, Zhang W, Mandal M, Meng MQ-H. Computer-aided bleeding detection in WCE video. IEEE J Biomed Health Inform 2014;18(2):636-42.

8. Amir-Khalili A, Peyrat J-M, Abinahed J, Al-Alao O, Al-Ansari A, Hamarneh G, et al. Auto localization and segmentation of occluded vessels in robot-assisted partial nephrectomy. Med Image Comput Comput Assist Interv 2014;17(1):407-14.

9. Chen H, L. Varshney LR, Varshney PK. Noise-Enhanced Information Systems. Proc IEEE 2014;102(10):1607-21.

10. Liu Y, Xu L, Zhu H, Liu SS-Y. Technical procedures for template-guided surgery for mandibular reconstruction based on digital design and manufacturing. Biomed Eng Online 2014;13:63.

11. Scuderi GR, Fallaha M, Masse V, Lavigne P, Amiot L-P, Berthiaume M-J. Total knee arthroplasty with a novel navigation system within the surgical field. Orthop Clin North Am 2014;45(2):167-73.

12. Quellec G, Charrière K, Lamard M, Droueche Z, Roux C, Cochener B, and G. Cazuguelet al. Real-time recognition of surgical tasks in eye surgery videos. Med Image Anal 2014;18(3):579-90.

13. Pheiffer TS, Thompson RC, Rucker DC, Simpson AL, Miga MI. Model-based correction of tissue compression for tracked ultrasound in soft tissue image-guided surgery. Ultrasound Med Biol $2014 \mathrm{Apr} ; 40(4): 788-803$

Correspondence to:

Sandrine Voros

Laboratoire TIMC-IMAG, équipe GMCAO

IN3S, pavillon Taillefer

Faculté de Médecine

38706 La Tronche Cedex, France

Tel: +33456520009

Fax +33456520055

E-mail: Sandrine.Voros@imag.fr

Appendix: Content Summaries of the Selected Best Papers for the IMIA Yearbook 2015, Section Sensors, Signal and Imaging Informatics

\section{Amir-Khalili A, Peyrat J-M, Abinahed J, Al-Alao 0, Al-Ansari A, Hamarneh G, Abugharbieh $\mathrm{R}$ \\ Auto localization and segmentation of occluded vessels in robot-assisted partial nephrectomy \\ Med Image Comput Comput Assist Interv 2014;17(1):407-14}

During a partial nephrectomy, the Hilar dissection stage consists in removing connective tissue that surround the renal artery and the renal vein. It is challenging because of the variability in patient vasculature and because vessels occluded by fat can be missed and inappropriately clamped, potentially leading to surgical complications. The authors propose an automatic segmentation approach to localize and label occluded vasculature on video images, based on phasebased video magnification. Phase-based video magnification reveals imperceptible motion from periodic changes in local phase information in images. The approach was evaluated retrospectively on eight real robot-assisted partial nephrectomies, by comparing the automatic segmentation with a semi-manually generated 3D model of the surgical environment. Renal artery and vein were distinguished because their pulsations are not in phase. The approach seems very encouraging although it requires that the surgical scene (including surgical instruments) is relatively fixed. The computation is not yet real-time ( 65 seconds on Matlab), but could be optimized.

\section{Mi H, Petitjean C, Dubray B, Vera P, Ruan S \\ Prediction of lung tumor evolution during radiotherapy in individual patients with PET \\ IEEE Trans Med Imaging 2014 \\ Apr;33(4):995-1003}

One of the main challenge for patients with pulmonary tumors is to be able to estimate the evolution of their tumors during radiotherapy in order to optimize the care process. Such an approach is now feasible through personalized modeling taking into account, not only morphological considerations but also functional considerations deducted from positron emission tomography imaging modality (TEP). In recent years, approaches for lung tumor growth modeling based on partial differential equations (PDE) modeling, and more specifically in the Advection Diffusion Reaction model have become increasingly popular: authors report an enrichment by taking into account the effect of radiotherapy (a treatment term is introduced in the cost function), by estimating the advective velocity field based on the estimation of the optical flow between two consecutive PET images, and by estimating the tumor growth model parameters using a volume dose-constraint (exponential decrease of the volume treated with respect to the radiation dose). The feasibility of such an approach has been evaluated using synthetic dataset with relevant performance under different levels of noise, and on clinical data coming from seven patients with non-small cell lung carcinoma: with only two consecutive temporal observations, the whole set of the modeling parameters is computed, enabling to estimate over time pulmonary tumor volume, with an accuracy compatible with a daily clinical practice.

\section{Pheiffer TS, Thompson RC, Rucker DC, Simpson AL, Miga MI}

Model-based correction of tissue compression for tracked ultrasound in soft tissue imageguided surgery

Ultrasound Med Biol 2014 Apr;40(4):788-803 
During ultrasound imaging, the ultrasound probe compresses the tissue, which impacts the quality of the registration of pre-operative information with intra-operative data. The authors present a method that reduces the error remaining after an initial rigid registration of the intra-operative ultrasound data and a pre-operative volumetric model: probe tracking information is used in combination with the co-registered tomograms to compute the distance between the probe surface and the pre-operative model surface. Two approaches were compared to estimate this distance: naive estimation of the translation, and estimation of the 3D displacements based on a Finite Elements biomechanical model. Based on this computed distance, the ultrasound images are then transformed to compensate for the compression, thus reducing the registration errors with the structures identified in pre-operative images (CT or MRI). The proposed method was validated through phantom experiments and a clinical case, and offers interesting perspectives for image-guided therapy.

\section{Quellec G, Charrière K, Lamard M, Droueche Z, Roux C, Cochener B, Cazuguel G Real-time recognition of surgical tasks in eye surgery videos}

Med Image Anal 2014 Apr;18(3):579-90
The authors present an automatic video-analysis method based on Content-Based Video Retrieval (CBVR) for the real-time recognition of surgical tasks in eye surgeries. Short video subsequences are characterized by a temporal array of instant feature vectors, each combining simple image characteristics (texture and color, motion). The originality of the approach lies in the way these instant feature vectors are combined into a fixed-length vector, independent of duration variations in the execution of the surgical task, allowing for a fast recognition rate. The method was evaluated on a dataset of retinal surgeries, a dataset of cataract surgeries, and a dataset of movie clips, with an overall accuracy ranging from 72 to $87 \%$ (best score for retinal surgery), with a framerate superior to 25 frames per second. A perspective of this work is the capacity to provide recommendations to the less experienced surgeons during surgical interventions.

\section{Tanter M, Fink M \\ Ultrafast imaging in biomedical ultrasound \\ IEEE Trans Ultrason Ferroelectr Freq Control 2014 Jan;61(1):102-19}

The authors performed a review focused on ultrafast imaging involving ultrasound technology, the ultrafast term being defined as any acquisition sequence enabling 2-D or 3-D imaging over a large field of view with a frame rate in the kilohertz range. On the contrary to conventional ultrasound imaging, the overall concepts associated to ultrafast ultrasound imaging consist in transmitting a wide field-of-view beam into the medium, recording the resulting backscattered echoes and finally performing digital parallel beamforming of the echoes to computationally build the final ultrasonic image from a single transmission. Among the advantages associated to the breakthrough of such imaging technology, and in comparison with conventional ultrasound approaches, two are mainly highlighted: a much higher amplitude signal thanks to the possibility to transmit a plane wave on the whole array aperture, a construction of high quality ultrasonic images because of an insonification of the whole region of interest within a very short period of time (hundreds of microseconds). With the maturity of this technology along with the progress in hardware and software computing technologies, real-time processing of ultrafast imaging is now ready to be achieved with exciting clinical imaging application such as Shear Wave Elastography, Ultrafast Doppler Imaging, Ultrafast Contrast Imaging and Functional Ultrasound Imaging on Brain activity. 